\title{
Biochemical analysis of heterotopic ossification in spinal cord injury patients
}

\author{
A Chantraine $^{1}$, B Nusgens ${ }^{2}$ and CM Lapiere ${ }^{2}$ \\ ${ }^{I}$ Division de Médecine Physique et de Rééducation, Hôpital Cantonal Universitaire, Beau-Séjour, 1211 Genève \\ 14. Switzerland; ${ }^{2}$ Laboratoire de Dermatologie Expérimentale, Institut de Pathologie, 4000 Sart Tilman, Liège, \\ Belgium
}

\begin{abstract}
Heterotopic ossification (HO) represents a frequent complication in spinal cord injury (SCI) patients. Samples of HO taken from SCI patients were studied and compared to normal bone. We used a procedure of bone particle fractionation (according to their degree of mineralisation) which allowed us to establish a profile reflecting the metabolic remodelling of bone and to analyse the organic matrix of the newly synthesised tissue. In paraplegic patients, we noted that there was a large increase of the proportion of a degree of calcified bone in the $\mathrm{HO}$ as we had previously observed in cortical as well as in cancellous bone of the same patients. Based on aminoacid analyses, we observed in the newly synthesised organic matrix of $\mathrm{HO}$ a decreased proportion of hydroxyprolyl residues resulting either from an alteration of the prolyl hydroxylation or from the presence of an excess of non-collagen polypeptides. These results are similar to those seen in sublesional bone of the SCI patients. This study demonstrates that HO is a newly formed bone which has a high rate of turnover as is seen in growing bone. This must be taken into account for the treatment of the patients.
\end{abstract}

Keywords: heterotopic ossification; spinal cord injury; biochemical analysis

\section{Introduction}

Heterotopic ossification (HO) in spinal cord injury patients, also called paraosteoarthropathies (POA), was described in 1918 by Dejerine and Ceillier ${ }^{1}$ from observations on spinal cord injury patients (SCI) during the first world war. They introduced the term POA (paraosteoarthropathy) although some other terms have been suggested, such as neurogenic osteoma, ossifying myositis in paraplegic patients, and heterotopic ossification, in fact the condition is neither an arthropathy nor a myositis but is really abnormally located ossification.

In SCI patients besides the $\mathrm{HO}$, bone metabolism is characterised by sublesional osteoporosis (occurring caudal to the neurological lesion). It is so striking that it may be observed by conventional radiographic techniques as early as 6 weeks after the SCI. It has been observed furthermore that urinary hydroxyproline is largely increased in those with paraplegia, suggesting that bone remodelling is stimulated. ${ }^{2,3}$ The urine hydroxyproline level increases much more when $\mathrm{HO}$ occurs. ${ }^{4}$

Besides histomorphometric analysis ${ }^{5}$ and calcium kinetics and scintigraphic studies (which has enabled us to study $\mathrm{HO}$ and to detect skeletal metabolic hyperactivity in paraplegic patient sublesional osteoporosis $)^{4}$ we now report on the biochemical analysis of $\mathrm{HO}$, collected by the total removal of $\mathrm{HO}$ from such patients. For this purpose we used a procedure of bone particles fractionation (according to their degree of mineralisation) which allowed us to establish a profile reflecting the metabolic remodelling of bone. ${ }^{6}$ In other words this technique enables us to know the extent of calcification of the matrix as performed in bone-the faster the remodelling, the greater is the proportion of less mineralised fractions, as seen during growth. ${ }^{6}$ These fractions were analysed to search for potential alterations of the protein matrix displayed modifications supporting an increased turnover of mineralised tissue and perhaps a defective post-translational processing of collagen that could account for increased amount of collagen breakdown products in the urine.

\section{Patients and methods}

\section{Patients}

The material was obtained from the total surgical removal of $\mathrm{HO}$ in five SCI patients, four men and one woman, 27-41 years old. All the samples were taken from $\mathrm{HO}$ between 12 and 16 months after the SCI, being located around the hip joint region. In parallel, we investigated a control group of seven samples collected from the iliac crest of seven men, aged 23-49 years old. All of them had either had a violent death or 
had died after an acute infection causing death in less than $48 \mathrm{~h}$. Autopsy excluded the possibility of disease which could affect bone metabolism.

\section{Fractionation of bone particles according to density} The samples of calcified tissues were completely cleaned of adhering tissues (muscles, tendons, ligaments). They were coarsely ground and were dehydrated by lyophilisation. Delipidation was performed on lyophilized fragments by repeated washings with petroleum benzin. The fragments were reduced into fine particles by crushing them in a stainless steel mortar until the whole sample could pass through a 375 mesh sieve. The maximum size of the particles, 40 microns, therefore represented a very small part of an osteone.

A continuous gradient of density (d), from 1.40 $\mathrm{g} \mathrm{cm}^{-3}$ to $2.44 \mathrm{~g} \mathrm{~cm}^{-3}$ was performed in a centrifuge tube by mixing toluene-bromoform mixtures as has been described earlier. ${ }^{7}$

$100 \mathrm{mg}$ powder was deposited through a sieve on the top of the organic gradient and covered with a $3 \mathrm{~mm}$ layer of distilled water. The tubes were centrifuged for $30 \mathrm{~min}$ at $23.000 \mathrm{~g}$ in a swinging buckets rotor SW 25 (Spinco) at $4{ }^{\circ} \mathrm{C}$. After centrifugation, the material floating at the junction between the layer of water and the organic solvent was collected (fraction 0 $<1.41 \mathrm{~g} \mathrm{~cm}^{-3}$ ) and the particles suspended in the organic gradient were collected by aspiration from the top of the tube in four successive fractions presenting progressively increasing density. The mean density of each of these four fractions measured by weighting a known volume of the liquid was $1.65 \mathrm{~g} \mathrm{~cm}^{-3}$ (fraction 1), $1.92 \mathrm{~g} \mathrm{~cm}^{-3}$ (fraction 2) $2.08 \mathrm{~g} \mathrm{~cm}^{-3}$ (fraction 3) and $2.17 \mathrm{~g} \mathrm{~cm}^{-3}$ (fraction 4 ). The particles were recovered from the organic liquid by centrifugation after adding a large amount of alcohol-ether (95-5 V/V). After two washings with the same solvent, the samples were dried overnight under vacuum in a dessicator and then weighed. The distribution of particles in each fraction was calculated as the percentage of total recovered particles. The recovery was $95.8 \pm 3.2 \%$.

Chemical composition of the bone organic matrix Weighted aliquots of the fractions $(0-4)$ large enough for analysis were hydrolysed for $24 \mathrm{~h}$ at $108^{\circ} \mathrm{C}$ in redistilled $5.6 \mathrm{~N} \mathrm{HCl}$ under a nitrogen atmosphere. The aminoacid analysis was performed in an automated aminoacid analyser (Beckman). The amount of organic material in the fraction was calculated from the aminoacid analysis. The proportion of collagen and non-collagen polypeptides (NCP) in the organic matrix was computed for each fraction assuming that collagen type I contains 100 residues of hydroxyproline per 1000 as described by Lapiere and Nusgens. ${ }^{6}$ The results were expressed in $\mathrm{mg}$ collagen and NCP per unit volume $\left(\mathrm{cm}^{3}\right)$. The calcium content of each fraction was determined using atomic absorption (Perkin-Elmer) and the phosphorus according to Eastoe. ${ }^{8}$

\section{Results}

Fractionation of bone and $\mathrm{HO}$ particles according to their degree of mineralisation

The distribution profiles of the particles fractionated according to density (fraction 0-4) from cancellous and cortical bone from iliac crest in normal subjects and from $\mathrm{HO}$ of paraplegic patients are illustrated in Figures 1 and 2 .

In cortical and cancellous bone collected from the iliac crest of control subjects (Figure 1) $55.1 \%$ and $49.8 \%$, respectively, of the particles sedimented in fraction 3 (mean density: $2.08 \mathrm{~g} \mathrm{~cm}^{-3}$ ), 39.9 and $43.3 \%$ in fraction 2 (mean density: $1.92 \mathrm{~g} \mathrm{~cm}^{-3}$ ). There was no significant difference between the values measured in the youngest control (23 years) as compared to the oldest (49 years) (not illustrated). In $\mathrm{HO}$ of SCI patients, we observed a striking increase in the proportion of particles sedimenting in fraction 2 as well as in the least calcified fractions ( 0 and 1$)$. The increase of this fraction in $\mathrm{HO}$ is highly significant compared to cortical as well as to cancellous bone of the control subjects $(P<0.001$ by Student's $t$ test $)$.

The calcium and phosphorus content was determined in each fraction from normal bone and HO. They steadily increased from fraction 0 to fraction 3 and for a given fraction displayed similar values in normal bone and $\mathrm{HO}$, as is illustrated in Table 1.

\section{Chemical composition of the organic matrix of the fractions}

The aminoacid composition of the organic matrix of the two largest fractions (2 and 3 ) in normal and $\mathrm{HO}$ were compared. The most representative aminoacids are quoted in Table 2 . There exists a significant reduction mainly in fraction 3 of $\mathrm{HO}$ in the proportion of hydroxyproline. The hydroxylysine and the glycine

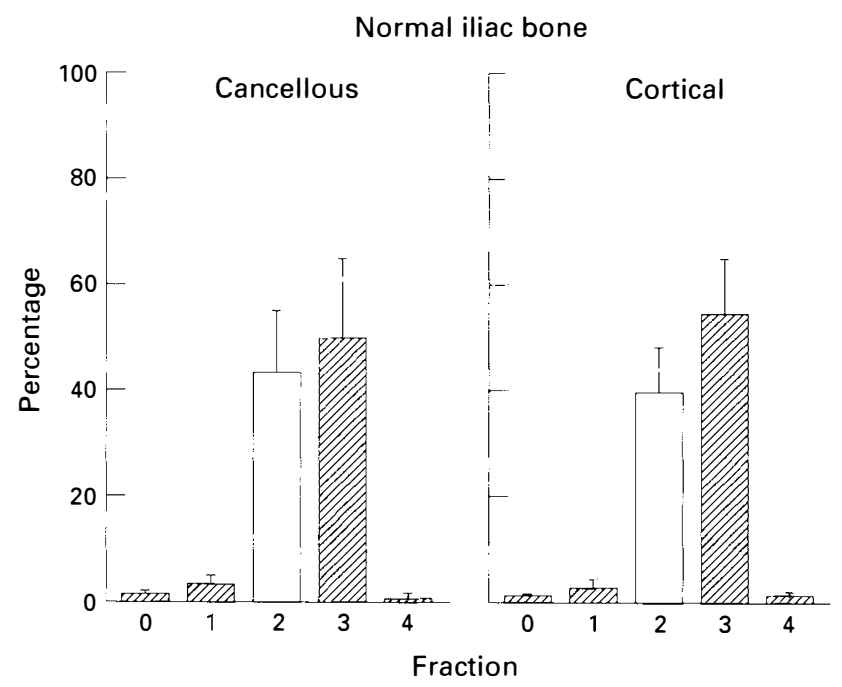

Figure 1 Distribution profile of the particles fractionated according to density (fraction 0-4) from cancellous and cortical iliac bone in normal subjects (control group). Particles sedimented in fraction 2 are respectively $43.3 \%$ and $49.8 \% ; 39.9 \%$ and $55.1 \%$ in fraction 3 


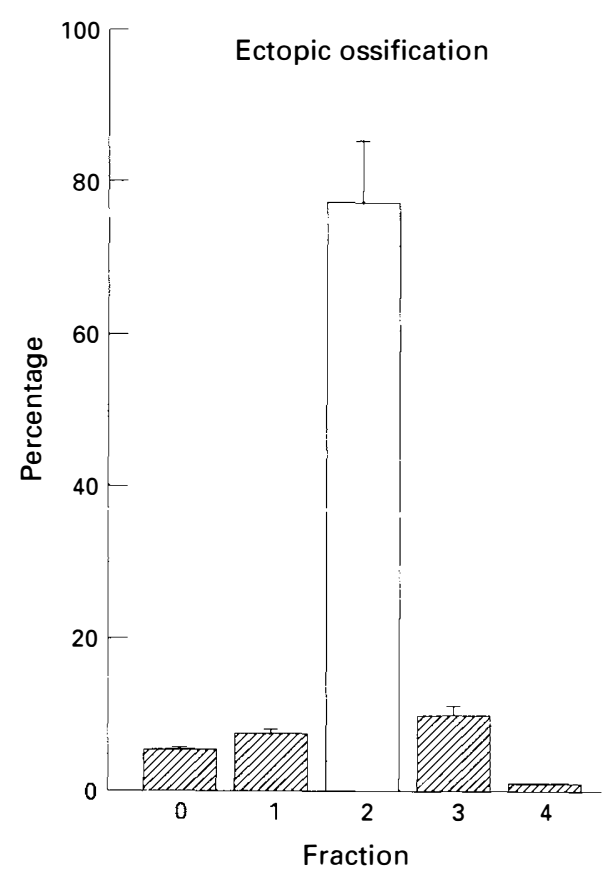

Figure 2 Distribution profile of the particles fractionated from $\mathrm{HO}$ of five SCI patients. There is a stricking increase in the proportion of particles sedimenting in fraction 2 compared to cortical and cancellous bone of the control subjects $(P<0.001$ Student's $t$ test $)$

Table 1 Mineral contents in bone $\left(\mathrm{mg} \mathrm{cm}^{-3}\right)$

\begin{tabular}{lcc}
\hline Fraction & Normal iliac bone & $H O$ \\
\hline Calcium & & \\
0 & $37 \pm 3$ & $40 \pm 0$ \\
1 & $141 \pm 1$ & $137 \pm 22$ \\
2 & $186 \pm 16$ & $215 \pm 10$ \\
3 & $213 \pm 22$ & $236 \pm 9$ \\
4 & $199 \pm 20$ & $142 \pm 40$ \\
Phosphates & & \\
0 & $21 \pm 1$ & $28 \pm 0$ \\
1 & $66 \pm 1$ & $67 \pm 8$ \\
2 & $89 \pm 12$ & $94 \pm 4$ \\
3 & $97 \pm 5$ & $113 \pm 13$ \\
4 & $81 \pm 10$ & $69 \pm 18$ \\
\hline
\end{tabular}

content are slightly modified while in most samples proline is significantly higher in the $\mathrm{HO}$.

The concentration of collagen per unit volume of the fraction (in $\mathrm{mg} \mathrm{cm}^{-3}$ ) in the two largest fractions ( 2 and 3 ) is significantly lower while the amount of NCP is significantly higher in the $\mathrm{HO}$ of paraplegic patients as compared to that of the control subjects for iliac bone.

\section{Discussion}

Regardless of its microarchitecture (osteone or lamellar) bone is composed of a mosaic of units presenting various degrees of mineralisation. We have previously demonstrated that during the process of bone calcification, the progressive deposition of mineral salts onto an organic matrix made of collagen and NCP is irreversible and proceeds at a similar rate independently of the age of the subject. ${ }^{7}$ The degree of mineralisation of an osteone is related to the biological age of the structure. ${ }^{6}$ The fractionation of bone particles according to their charge in minerals allows us to obtain a profile of distribution which is therefore related to the metabolic activity of the tissue. A high rate of turnover, as occurs during growth, will be associated with a high proportion of the least calcified fractions. ${ }^{6}$ Adult normal human bone is composed of a large proportion of fully calcified matrix as was illustrated for the control subjects of this study.

The HO studied in our paraplegic patients presented a distribution profile characterised by an increased proportion of less mineralised particles. This supports the view that the $\mathrm{HO}$ in those with paraplegia is a newly formed tissue with a high rate of turnover, which was also demonstrated in a previous study in sublesional bones in SCI patients, ${ }^{9}$ and which is also seen in other osteoporotic conditions. ${ }^{10,11}$ A marked increase in the urinary excretion of hydroxyproline $\mathrm{e}^{2,3}$ and some parameters of $45 \mathrm{Ca}$ kinetic studies ${ }^{5}$ agree with this hypothesis. Furthermore, we recently showed that the osteocalcine which represents an index of bone formation is increased in SCI patients and mostly in patients with $\mathrm{HO} .{ }^{12}$ This has been confirmed in a subsequent study. ${ }^{13}$ Moreover an interesting published work showed that serum from paraplegic patients with $\mathrm{HO}$ incubated with human osteoblasts in tissue culture produces significantly greater levels of osteoblasts stimulating factors than does serum from paraplegics without $\mathrm{HO} .^{14}$

The aminoacid composition of the organic matrix of the fraction 2 from normal bone demonstrates that it is composed for $90 \%$ of collagen as calculated on the basis of hydroxyproline. In SCI patients with $\mathrm{HO}$, we observed a decreased proportion of hydroxyproline residues as we previously also described for sublesional bone. ${ }^{9}$ This defect could result from a reduced hydroxylation of proline or could depend on an accumulation of NCP. In the former situation we should have observed an increase in the number of proline residues equal to the reduction of hydroxyproline. The increase is only of five proline residues instead of the expected 10. As the thermal stability of the triple helix of collagen is known to depend on the degree of hydroxylation of the proline, ${ }^{15}$ a defective prolylhydroxylation could account for a higher susceptibility to degradation of the bone matrix in paraplegic patients. The presence of an excess of NCP in each fraction in paraplegic bone could also explain the increase in some aminoacids present in a large proportion in glycoproteins (as leucine), and the smaller than expected increase in proline. The calculated mean NCP is significantly higher in the bone of paraplegic patients than it is in normal subjects. ${ }^{9}$

In conclusion, the study of the $\mathrm{HO}$ of SCI patients by centrifugation of density gradient and by chemical analysis of the organic matrix shows that $\mathrm{HO}$ is a newly formed bone not yet completely mineralised. It also 
Table 2 The aminoacid composition of the protein matrix of fractions 2 and 3 from normal iliac bone (Normal) and heterotopic ossification (HO) from spinal cord injury patients is expressed in number of residues per thousand quoted for the most representative aminoacids

\begin{tabular}{|c|c|c|c|c|}
\hline & \multicolumn{2}{|c|}{ Fraction 2} & \multicolumn{2}{|c|}{ Fraction 3} \\
\hline & $\begin{array}{c}\text { Normal } \\
\text { (7) }\end{array}$ & $\begin{array}{c}\mathrm{HO} \\
(5)\end{array}$ & $\begin{array}{c}\text { Normal } \\
\text { (6) }\end{array}$ & $\begin{array}{c}\mathrm{HO} \\
(5)\end{array}$ \\
\hline $\mathrm{OH}$ proline & $89 \pm 2.0$ & $84^{*} \pm 3.7$ & $93 \pm 0.5$ & $84^{* *} \pm 6$ \\
\hline Proline & $112 \pm 4.1$ & $118^{*} \pm 2$ & $116 \pm 0.8$ & $120 \pm 1.4$ \\
\hline OH lysine & $3.5 \pm 0.4$ & $3.8 \pm 0.4$ & $3.8 \pm 0.3$ & $4.0 \pm 0.8$ \\
\hline Lysine & $32 \pm 1.75$ & $30 \pm 2.5$ & $30 \pm 2.8$ & $28 \pm 3.4$ \\
\hline Glycine & $302 \pm 7.5$ & $305 \pm 8.3$ & $315 \pm 4$ & $316 \pm 8$ \\
\hline Glutamic acid & $80 \pm 1.3$ & $78 \pm 3$ & $77 \pm 0.5$ & $78 \pm 4$ \\
\hline Leucine & $31 \pm 1.5$ & $33 \pm 3$ & $29 \pm 0.25$ & $32 \pm 3.2$ \\
\hline$\%$ Hydroxylation proline & 44.4 & $41.5^{* *}$ & 44.2 & $41.2^{* *}$ \\
\hline
\end{tabular}

${ }^{*} P<0.05 ;{ }^{* *} P<0.01$

suggests that $\mathrm{HO}$ has a high rate of turnover as is seen in growing bone, a finding confirmed by the increase of osteocalcine during the life of paraplegic people.

\section{References}

1 Dejerine Mme, Ceillier A. Trois cas d'ostéomes-ossifications périostées juxta-musculaires et interfasciculaires chez des paraplégiques par lésion traumatique de la moelle épinière. Rev Neurol 1918; 33: 156.

2 Klein L, Van De Noort S, Dejak JJ. Sequential studies of urinary hydroxyproline and serum alakaline phosphatase in acute paraplegia. Med Serv J Can 1966; 22: 524-533.

3 Chantraine A. Clinical investigation of bone metabolism in spinal cord lesions. Paraplegia 1971; 8: 253-259.

4 Minaire $\mathrm{P}$ et al. Quantitative histological data on disuse osteoporosis. Calcif Tiss Res 1974; 17: 57-73.

5 Chantraine A. L'ostéoposrose et les Parostéoarthropathies au Cours de la Paraplégie. Editions Arscia: Bruxelles, 1979; p 238.

6 Lapiere ChM, Nusgens B. Maturation related changes of the protein matrix of bone. In: Balazs EA (ed). Chemistry and Molecular Biology of the Intercellular Matrix, Collagen, Basal Laminae Elastin. Academic Press: London and New York, 1978; pp 55-98.
7 Nusgens B, Chantraine A, Lapiere ChM. Protein in the matrix of bone. Clin Orthop 1972; 88: 252-274.

8 Eastoe JE. Methods for the determination of phosphate, calcium and protein in small portions of mineralized tissues. In: Richelle LJ, Dallemagne MJ (eds). Proceedings of the Second European Symposium on Calcified Tissues, 1965; pp 265-374.

9 Chantraine A, Nusgens B, Lapiere ChM. Bone remodeling during the development of osteoporosis in paraplegia. Calcif Tiss Int 1986; 38: 323-327.

10 Whyte MP et al. Postmenopausal osteoporosis. A heterogenous disorder as assessed by histomorphometric analysis of iliac crest bone from untreated patients. Am J Med 1982; 72: 193-202.

11 Van Ouwenaller C, Uebelhart D, Chantraine A. Bone metabolism in hemiplegic patients. Scand J Rehabil Med 1989; 21: $165-170$.

12 Zanone X, Castanier M, Chantraine A. Dosage de l'ostéocalcine chez les blessés médullaires. Réadaptation-Revalidatie 1991; 1-2: 89-93.

13 Mysiw WJ, Tan J, Jackson RD. Heterotopic ossification: the utility of osteocalcin in diagnosis and management. Am J Phys Med Rehabil 1993; 72: 184-187.

14 Kurer MHJ, Khoker MA, Dandona P. Human osteoblast stimulation by sera from paraplegic patients with heterotopic ossification. Paraplegia 1992; 30: 165-168.

15 Rosenbloom J, Harsch M, Jimenez S. Hydroxyproline content determines the denaturation temperature of chick tendon collagen. Arch Biochem Biophys 1973; 156: 478. 